\title{
In vitro and in vivo antiprotozoal activities of bispolides and their derivatives
}

\author{
The Journal of Antibiotics (2010) 63, 275-277; doi:10.1038/ja.2010.32; published online 9 April 2010
}

During the course of our screening program to discover new antiprotozoal (antimalarial and antitrypanosomal) chemicals, we have evaluated isolates from soil microorganisms, as well as compounds from the antibiotic libraries of the Kitasato Institute for Life Sciences and Nimura Genetic Solutions. We have previously reported various microbial metabolites exhibiting potent antimalarial ${ }^{1-4}$ and antitrypanosomal properties. ${ }^{5-7}$ We have recently found that the known 20-membered ring macrodiolide antibiotics, the bispolides, ${ }^{8}$ together with new derivatives, exhibit selective antitrypanosomal and potent antimalarial activities, both in vitro and in vivo. Here, we report the antitrypanosomal and antimalarial profiles of bispolides, their derivatives (Figure 1) and the related 16-membered ring macrodiolide antibiotic as elaiophylin (azalomycin B) ${ }^{9,10}$ (Figure 1) in comparison with those of clinically used antitrypanosomal drugs, such as suramin and eflornithine, and two clinically used antimalarial drugs, artemisinin and chloroquine. We also present some conclusions on structure-activity relationships.

Bispolides A1, A3, B1 and B3 were purified from the culture broth of Microbispora sp. A34030. ${ }^{8}$ Derivatives of bispolide A1 (derivatives I and II) were prepared as follows. The 13,13'-dimethoxy compound, bispolide A3, prepared from bispolide A1 by reaction with $0.04 \%-\mathrm{HCl}$ in $\mathrm{MeOH}$, was reduced by $\mathrm{NaBH}_{3} \mathrm{CN}$ in EtOH to give the 13,13'dideoxy compound (derivative I (ESI-MS $m / z$ 1123.69114; $\left.{ }^{12} \mathrm{C}_{62}{ }^{1} \mathrm{H}_{100}{ }^{23} \mathrm{Na}_{1}{ }^{16} \mathrm{O}_{16}\right)$ ). Partial hydrolysis of derivative $\mathrm{I}$ in the presence of $p$-toluenesulfonic acid in aqueous acetonitrile gave derivative II (FAB-MS $\mathrm{m} / \mathrm{z} 841$ $\left(\mathrm{M}^{+}\right)$). Elaiophylin was obtained from the antibiotic library of the Kitasato Institute for Life Sciences.

In vitro antiprotozoal activities against Trypanosoma brucei brucei strain GUTat 3.1, Plasmodium falciparum strains K1 (drugresistant) and FCR3 (drug-sensitive), and cytotoxicity against human diploid embryonic cell line MRC-5 were measured as described previously. ${ }^{1,5}$ In vivo antitrypanosomal activity for T. b. brucei strain S427 was measured as described previously. ${ }^{6}$ Test compounds were solubilized in an aqueous mixture of $10 \%$ DMSO-Tween 80 and $\mathrm{EtOH}$ (7:3) and administered i.p. to mice on the next day (day 1) following infection with parasites (day 0). Subsequently, the compounds were successively administered (i.p.) to the infected mice once a day for 3 days (days 2-4). Efficacies of compounds were determined by the parasitemia levels and the mean of survival days (MSD), compared with that of the untreated control mice.

Table 1 shows the in vitro antiprotozoal activities of bispolides, their derivatives, elaiophylin and some standard antiprotozoal drugs. Bispolides, derivative I (13,13'-dideoxybispolide A1) and elaiophylin showed the more potent antimalarial activity against the drug-resistant $\mathrm{K} 1$ strain of $P$. falciparum, in the $260-620 \mathrm{ng} \mathrm{ml}^{-1}$ range. The half-maximal inhibitory concentration $\left(\mathrm{IC}_{50}\right.$ ) values against the drug-sensitive FCR3 strain of $P$. falciparum were similar to those against the $\mathrm{K} 1$ strain of $P$. falciparum (data not shown). The antimalarial activity was similar to that of chloroquine, but 43-103-fold less than that of artemisinin. However, the antimalarial activity of derivative II was 14 -fold less than that of bispolide A3.

With respect to antitrypanosomal activity, bispolides and derivative I showed the most potency against the GUTat 3.1 strain of T. $b$. brucei, in the $57-150 \mathrm{ng} \mathrm{ml}^{-1}$ range. The antitrypanosomal activities were 10-40-fold more potent than that of the standard drugs, suramin and eflornithine. However, the antitrypanosomal activities of derivative II and elaiophylin were 3-6-fold less than that of bispolide A3.

The in vitro cytotoxicities of bispolides, their derivatives, elaiophylin and some stan- dard antiprotozoal drugs are presented in Table 1. Among them, the $\mathrm{IC}_{50}$ values of bispolide $\mathrm{B} 1$ and elaiophylin were 0.96 and $0.87 \mu \mathrm{g} \mathrm{ml}^{-1}$, respectively. The $\mathrm{IC}_{50}$ values of bispolide $\mathrm{A} 1, \mathrm{~A} 3$ and $\mathrm{B} 3$, and derivative I were in the range of $1.4-3.9 \mu \mathrm{g} \mathrm{ml}^{-1}$, whereas that of derivative II was $15.6 \mu \mathrm{g} \mathrm{ml}^{-1}$. To compare the antiprotozoal activities and cytotoxicities, we introduced selectivity indexes (SIs: cytotoxicity $\left(\mathrm{IC}_{50}\right.$ for the MRC-5 cells)/antimalarial or antitrypanosomal activity $\left(\mathrm{IC}_{50}\right.$ for the $\mathrm{K} 1$ strain or the GUTat 3.1 strain), as presented in Table 1. In the case of the MRC-5 cells/K1 strain, bispolide A1 showed a medium SI, with a ratio of 15. Other bispolides and elaiophylin showed a low SI, with ratios of 2-9. In the case of the MRC- 5 cells/GUTat 3.1 strain, bispolides A1 and B3 showed a moderate SI, with ratios of 57-62. Other bispolides and elaiophylin showed a medium or low SI (ratios of 2-26). Among the bispolides, we were interested in bispolides A3 and B3, which had an SI of 26 and 62, respectively. Among the tested bispolides, B3 showed the more potent antitrypanosomal activity and highest SI, whereas bispolide A3 exhibited potent antitrypanosomal activity but with medium SI.

The preliminary in vivo antitrypanosomal activities of bispolides A3 and B3 were measured in the T. $b$. brucei S-427 acute mouse model. At a dose of $25 \mathrm{mg} \mathrm{kg}^{-1}$, bispolide B3 did not achieve cure but did extend the MSD to 9.5 days, representing a 1.4 -fold increase over control MSD (7.0 days). The same dose of bispolide A3 did not achieve cure but also extended the MSD. Under the same conditions, suramin showed a curative effect (MSD: $>30$ days) at a dose of $1 \mathrm{mg} \mathrm{kg}^{-1}$. The bispolide B3 data suggest that it is possibly a new candidate compound for discovering new antitrypanosomal drugs with more potent activity. These efficacy tests, including in vivo antimalarial activity, are being investigated further. 


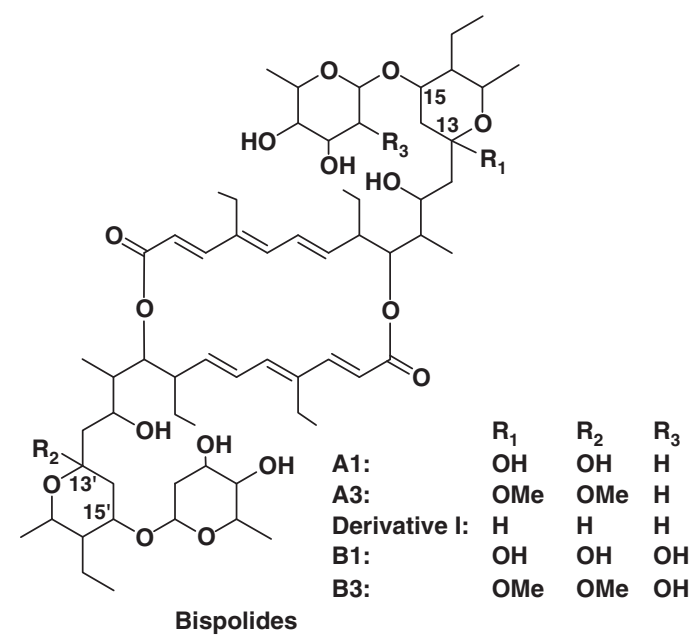

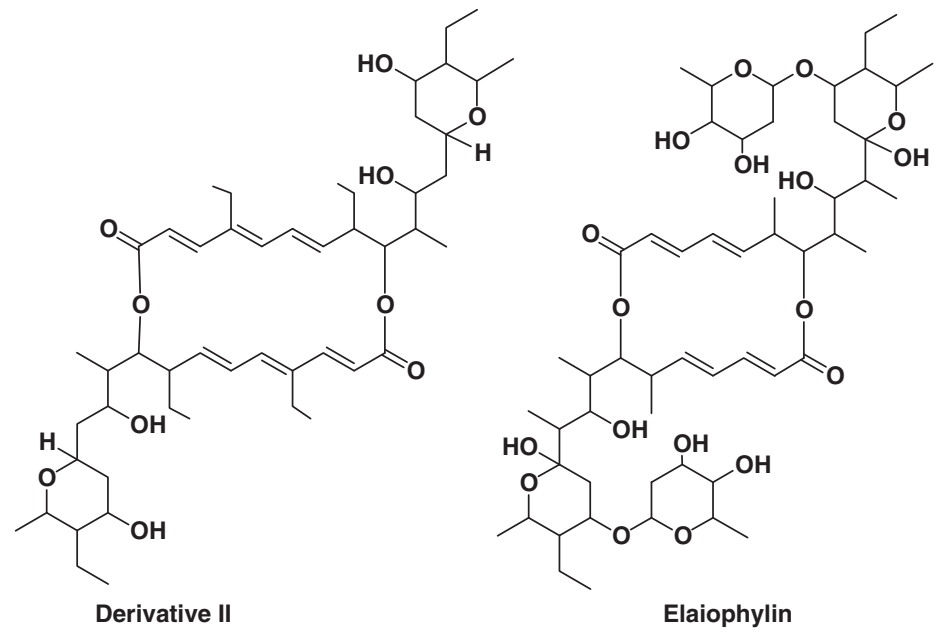

Figure 1 Structures of bispolides and elaiophylin.

The weak antiprotozoal activities of derivative II in comparison with bispolide A3 (the parent compound) and derivative I provide very interesting information on the structure-activity relationships. In contrast to bispolide A3 and derivative I, which possess hexose moieties at $15-\mathrm{OH}$ and $15^{\prime}-\mathrm{OH}$ of the aglycone macrodiolide, derivative II lacks the hexose moieties and is 5.8-14.3-fold less active for antiprotozoa than bispolide $\mathrm{A} 3$ and derivative I. Furthermore, bispolides A1, A3 and $\mathrm{B} 3$, together with derivative I, possess two conjugated trienes in the 20 -membered ring. Elaiophylin possesses two conjugated dienes in the 16-membered ring and has 3-8-fold less antitrypanosomal activity and 1.6-4.5-fold less potent cytotoxicity than bispolides A1, A3, B3 and derivative I. Our data therefore suggest that the hexose moieties at $15-\mathrm{OH}$ and $15^{\prime}-\mathrm{OH}$ and the two conjugated trienes in the 20 -membered ring present in bispolides have a significant antiprotozoal activity and cytotoxicity.

Further studies are necessary for an extensive structure-in vitro antiprotozoal activity evaluation of bispolide-related compounds.

We previously reported that bispolides have inhibitory activity against Gram-positive bacteria, including MRSA. ${ }^{8}$ Related 16-membered ring macrodiolide antibiotics, such as elaiophylin and its asymmetric deglycosidation products, have been reported to show both antibacterial and nematocidal traits. ${ }^{10}$ However, discovery of the selective antitrypanosomal activity and the potent antimalarial activity of bispolides is novel and our data constitute the first report of such properties.

The above results reveal that bispolides are promising lead compounds for development of novel antiprotozoal (antitrypanosomal and antimalarial) drugs. Further study of

Table 1 In vitro antiprotozoal activity against Plasmodium falciparum $\mathrm{K} 1^{\mathrm{a}}$ and Trypanosoma brucei brucei GUTat 3.1 strains, plus cytotoxicity in MRC-5 cells, of bispolides and their derivatives

\begin{tabular}{|c|c|c|c|c|c|}
\hline \multirow[b]{3}{*}{ Compound } & \multicolumn{3}{|c|}{$I_{50}\left(\mathrm{ng} \mathrm{m}^{-1}\right)$} & & \\
\hline & \multicolumn{2}{|c|}{ Antiprotozoal activity } & \multirow{2}{*}{$\begin{array}{c}\text { Cytotoxicity } \\
\text { MRC-5 }\end{array}$} & \multicolumn{2}{|c|}{ Selectivity index (SI) } \\
\hline & P. f. $K 1$ & T. b. b. GUTat 3.1 & & $M R C-5 / K 1$ & MRC-5/GUTat 3.1 \\
\hline Bispolide A1 & 260 & 67 & 3820 & 15 & 57 \\
\hline Bispolide A3 & 480 & 150 & 3910 & 8 & 26 \\
\hline Bispolide B1 & 390 & 57 & 960 & 3 & 17 \\
\hline Bispolide B3 & 450 & 63 & 3910 & 9 & 62 \\
\hline Derivative I & 620 & 120 & 1390 & 2 & 11 \\
\hline Derivative II & 6850 & 870 & 15630 & 2 & 18 \\
\hline Elaiophylin & 370 & 460 & 870 & 2 & 2 \\
\hline Artemisinin & 6 & ND & 45170 & 7528 & - \\
\hline Chloroquine & 184 & ND & 18572 & 101 & - \\
\hline Suramin & ND & 1580 & $>100000$ & - & $>63$ \\
\hline Eflornithine & ND & 2270 & $>100000$ & - & $>44$ \\
\hline
\end{tabular}

Abbreviations: IC50, half-maximal inhibitory concentration; ND, not determined. aDrug-resistant strain.

the antiprotozoal and other biological activities of bispolides is in progress.

\section{ACKNOWLEDGEMENTS}

This work was supported, in part, by funds from the Drugs for Neglected Diseases initiative (DNDi), Quality Assurance Framework of Higher Education from the Ministry of Education, Culture, Sports, Science and Technology, Japan (MEXT), Japan Science and Technology Agency (JST) and the All Kitasato Project Study (AKPS). We are grateful to Ms H Sekiguchi and Mr T Furusawa for their technical assistance.

Kazuhiko Otoguro $^{1}$, Masato Iwatsuki ${ }^{1}$, Aki Ishiyama ${ }^{1}$, Miyuki Namatame ${ }^{1}$, Aki Nishihara-Tsukashima ${ }^{1}$, Seigo Sato ${ }^{2}$, Masahiro Hatsu ${ }^{2}$, Hironobu Iinuma ${ }^{2}$,
Seiji Shibahara ${ }^{2}$, Satoshi Nimura ${ }^{2}$, Shinichi Kondo ${ }^{2}$, Haruki Yamada ${ }^{3}$ and Satoshi Ōmura ${ }^{3}$

${ }^{1}$ Research Center for Tropical Diseases, Kitasato Institute for Life Sciences, Kitasato University, Tokyo, Japan; ${ }^{2}$ Nimura Genetic Solutions, Tokyo, Japan and ${ }^{3}$ Kitasato Institute for Life Sciences,

Graduate School of Infection Control Sciences, Kitasato University, Tokyo, Japan E-mail: otoguro@lisci.kitasato-u.ac.jp or omuras@insti.kitasato-u.ac.jp

1 Otoguro, K. et al. Potent antimalarial activities of polyether antibiotic, X-206. J. Antibiot. 54, 658-663 (2001). 
2 Otoguro, K. et al. In vitro and in vivo antimalarial activities of a non-glycoside 18-membered macrolide antibiotic, borrelidin against drug-resistant strains of Plasmodia. J. Antibiot. 56, 727-729 (2003).

3 Otoguro, K. et al. In vitro and in vivo antimalarial activities of a carbohydrate antibiotic, prumycin, against drug-resistant strains of Plasmodia. J. Antibiot. 57, 400-402 (2004).

$4 \mathrm{Ui}, \mathrm{H}$. et al. Selective and potent in vitro antimalarial activities found in four microbial metabolites. J. Antibiot. 60, 220-222 (2007).
5 Otoguro, K. et al. Selective and potent in vitro antitrypanosomal activities of 10 microbial metabolites. J. Antibiot. 61, 372-378 (2008).

6 Ishiyama, A. et al. In vitro and in vivo antitrypanosomal activities of two microbial metabolites, KS-505a and Alazopeptin. J. Antibiot. 61, 627-632 (2008).

7 Ishiyama, A. et al. In vitro and in vivo antitrypanosomal activities of three peptide antibiotics: Leucinostatin $A$ and B, alamethicin I and tsushimycin. J. Antibiot. 62, 303-308 (2009).
8 Okujo, N. et al. Bispolides, novel 20-membered ring macrodiolide antibiotics from Microbispora. J. Antibiot. 60, 216-219 (2007).

9 Ōmura, S. in Macrolide Antibiotics, Chemistry, Biology and Practice; Production, Structure and Biological Properties of Macrolide-Like Antibiotics (ed. Ōmura, S.) 509-552 (Academic Press, Orlando, 1984).

10 Hammann, P., Kretzschmar, G. \& Seibert, G. Secondary metabolites by chemical screening. I. Elaiophylin derivatives and their biological activities. J. Antibiot. 43, 1431-1440 (1990). 